\title{
Effects of Susceptibility Artifacts on Perfusion MRI in Patients with Primary Brain Tumor: A Comparison of Arterial Spin-Labeling versus DSC
}

\author{
(D) H. Maral, DE. Ertekin, (D) Ö. Tunçyürek, and (D). Özsunar
}

\begin{abstract}
BACKGROUND AND PURPOSE: Our aim was to investigate the effects of intratumoral hemorrhage, calcification, and postoperative changes on the sensitivity of arterial spin-labeling and DSC perfusion MR imaging in patients with primary brain tumors.

MATERIALS AND METHODS: Eighty-six brain tumor lesions were examined with single-phase and multiphase arterial spin-labeling and DSC perfusion MR imaging. The lesions that had no intratumoral bleeding/calcifications and history of surgery were assigned to group 1 $(n=38)$, and the lesions that had these were assigned to group $2(n=48)$. The relative regional cerebral blood flow was calculated in both perfusion methods, and relative regional cerebral blood volume was calculated in DSC. Imaging results were correlated with histopathology or follow-up.

RESULTS: In the quantitative evaluation, the sensitivity and specificity of relative regional cerebral blood flow in multiphase arterial spin-labeling perfusion were $94.4 \%$ and $80 \%$ in group 1 and $78.3 \%$ and $88 \%$ in group 2 , respectively. The sensitivity and specificity of relative regional cerebral blood flow in DSC perfusion were $88.9 \%$ and $75 \%$ in group 1 and $78.3 \%$ and $84 \%$ in group 2 , respectively. The sensitivity and specificity of relative regional cerebral blood volume in DSC perfusion were $66.7 \%$ and $100 \%$ in group 1 and $69.6 \%$ and $96 \%$ in group 2, respectively. In the qualitative evaluation, the sensitivities for single-phase and multiphase arterial spin-labeling were $48.2 \%$ and $79.3 \%$, respectively, with $100 \%$ specificity for both.
\end{abstract}

CONCLUSIONS: The sensitivity and specificity of multiphase arterial spin-labeling were similar to those of DSC perfusion irrespective of bleeding and calcification in primary brain tumors. Thus, we suggest that noncontrast multiphase arterial spin-labeling can be used instead of DSC perfusion MR imaging in the diagnosis and follow-up of intracranial tumors.

ABBREVIATIONS: $\mathrm{ASL}=$ arterial spin-labeling; $\mathrm{mpASL}=$ multiphase $\mathrm{ASL} ; \mathrm{PI}=$ perfusion imaging; $\mathrm{rCBF}=$ relative $\mathrm{CBF}$; ROC = receiver operating characteristic; $r \mathrm{rCBF}=$ relative regional $\mathrm{CBF}$; $\operatorname{rrCBV}=$ relative regional $\mathrm{CBV}$; $\mathrm{sPASL}=$ single-phase $\mathrm{ASL}$

$\mathbf{P}$ erfusion MR imaging is a technique that provides information on angiogenesis at the microscopic level. ${ }^{1}$ DSC MR imaging is a widely used and generally accepted MR imaging perfusion method for brain tumor diagnosis and staging. ${ }^{2-4}$ Arterial spin-labeling (ASL) is a promising perfusion MR imaging method, which is repeatable, low-cost, and noninvasive and does not require the administration of exogenous contrast. ${ }^{5}$

Brain tumors are often heterogeneous lesions and may include bleeding and calcification. ${ }^{6}$ In addition, bleeding residues such as hemosiderin are frequently seen in patients postoperatively. ${ }^{2,7}$ It is

Received June 21, 2019; accepted after revision November 25.

From the Department of Radiology (H.M.), Dr. Siyami Ersek Thoracic and Cardiovascular Surgery Training and Research Hospital, Istanbul, Turkey; Department of Radiology (E.E., Ö.T., Y.Ö.), Aydın Adnan Menderes University Faculty of Medicine, Aydın, Turkey; and Department of Radiology (Ö.T.), Near East University Faculty of Medicine, Nicosia, Cyprus.

Please address correspondence to Hakan Maral, Dr. Siyami Ersek Thoracic and Cardiovascular Surgery Training and Research Hospital, Department of Radiology, 13 Tıbbıye Street, Üsküdar/İstanbul 34668, Turkey; e-mail: hknmaral@gmail.com http://dx.doi.org/10.3174/ajnr.A6384 known that the content of hemorrhage residues and calcium disturb the inhomogeneity of the magnetic field, reducing the sensitivity of perfusion by causing susceptibility artifacts. ${ }^{8}$ Some studies have reported that DSC perfusion imaging (PI) is more commonly affected by susceptibility artifacts compared with ASL-PI. ${ }^{9-11}$ However, there are no large case series studies investigating this issue systematically. The primary purpose of our study was to investigate how these artifacts affect the sensitivity of both perfusion techniques in distinguishing malignant from benign brain tumors. The second aim was to compare the sensitivity of single- and multiphase ASL (spASL and mpASL) methods in distinguishing malignant from benign brain tumors. In addition, we compared the relative regional $\mathrm{CBV}(\mathrm{rrCBV})$ and relative regional $\mathrm{CBF}(\mathrm{rrCBF})$ values used in the literature to determine which method was more useful.

\section{MATERIALS AND METHODS}

Approval of the local ethics committee was obtained for the study.

AJNR Am J Neuroradiol 41:255-61 Feb 2020 www.ajnr.org 


\begin{tabular}{lccc}
\hline \multicolumn{1}{c}{ Sequence Parameters } & spASL & mpASL & DSC \\
\hline Acquired orientation & Axial & Axial & Axial \\
TR (ms) & 4000 & 250 & 1800 \\
TE (ms) & 25 & 20 & 40 \\
Flip angle & $70^{\circ}$ & $35^{\circ}$ & $75^{\circ}$ \\
FOV (mm") & 240 & 240 & 224 \\
Matrix (pixel) & $68 \times 68$ & $68 \times 68$ & $88 \times 88$ \\
Thickness/gap (mm) & $6 / 0.6$ & $6 / 0.6$ & $5 / 0$ \\
Pulse delay times (ms) & 1200 & $300,550,800,1050,1300,1550,1800,2050$ & - \\
Label thickness/gap (mm) & $100 / 20$ & $130 / 20$ & - \\
Acquisition time & 4 min $8 \mathrm{sec}$ & $4 \mathrm{~min} 8 \mathrm{sec}$ & $1 \mathrm{~min} 40 \mathrm{sec}$ \\
\hline
\end{tabular}

Note:--indicates "no labelling" and "no pulse delay time"; DSC, dynamic susceptibility contrast; FOV, field-of-view; mpASL, multi phase arterial spin-labeling; spASL, single phase arterial spin-labeling; TE, echo time; TR, repetition time.

\section{Patient Selection}

Records of all lesions were retrospectively reviewed for patients who were diagnosed with an intracranial mass and who had undergone perfusion MR imaging at Adnan Menderes University, Department of Radiology, with both ASL-PI and DSC-PI methods between January 2011 and February 2017. In total, we investigated 133 patients. A total of 54 lesions were excluded from the study, consisting of 7 lesions with an extra-axial mass, 8 lesions that were thought to be benign but were lost to follow-up, 5 lesions in patients who died of nontumor causes, 9 lesions without primary brain tumor, and 25 lesions with unverified diagnosis. A total of 86 lesions from 79 patients were included in the study.

The diagnosis of the lesions was confirmed either histopathologically or by performing follow-up MR imaging for at least 11 months. In the follow-up period, the lesions were considered benign when they did not appear different in terms of size, appearance, and enhancement compared with the previous images. The lesions were considered malignant when progression was observed in the images during the follow-up period. Samples for histopathologic examination were obtained by an operation or stereotactic biopsy.

The grading of tumors was performed on the basis of the pathologic criteria of the World Health Organization (2016) (grades I, II, III, IV). Grade I and II tumors were considered lowgrade (benign), whereas grade III and IV tumors were considered high-grade (malignant).

\section{Imaging Protocol}

MR imaging was performed at 1.5T (Achieva; Philips Healthcare, Best, the Netherlands). T1-weighted axial, T2-weighted axial, FLAIR axial, and T2-weighted sagittal MR images were obtained during conventional brain $\mathrm{MR}$ imaging. The $\mathrm{T} 2{ }^{*}$ sequence was used to determine intralesional bleeding or calcifications. The separation of calcium and blood from each other cannot be done using only the $\mathrm{T} 2{ }^{*}$ sequence. We did not make such a distinction. The ASL perfusion sequence was performed before administering the MR imaging contrast agent. The pulsed ASL technique was used for spASL and mpASL. Afterward, a DSC sequence was performed using an automatic injector. Gadoterate meglumine was administered as the contrast agent via the antecubital vein at a dose of $0.1 \mathrm{mmol} / \mathrm{kg}(1 \mathrm{mmol}=0.5 \mathrm{~mL})$ using an 18 -ga IV catheter at a rate of $10 \mathrm{~mL} / \mathrm{s}$ automatically (Spectris Solaris EP MR Injection System; MedRad, Indianola, Pennsylvania). This was followed by $20 \mathrm{~mL}$ of physiologic saline solution injection at almost the same rate. Parameters of the conventional brain MR imaging sequence were the following: T1-weighted, matrix $=212 \times 168$ (T2weighted $=232 \times 168) ; \mathrm{FOV}=210 \mathrm{~mm}$; TR $=460 \mathrm{~ms}(\mathrm{~T} 2$ weighted $=5700 \mathrm{~ms}) ; \mathrm{TE}=10 \mathrm{~ms}(\mathrm{~T} 2$-weighted $=110 \mathrm{~ms})$; flip angle $=69^{\circ}\left(\mathrm{T} 2\right.$-weighted $\left.=90^{\circ}\right)$; thickness $=5 \mathrm{~mm}$ with 1-mm interslice gaps; number of slices $=23$; acquisition time $=90$ seconds.

Multiphase ASL was capable of multisection image acquisition at multiple TI (inversion time) points and was based on the pulsed ASL technique. Image acquisition was performed at 8 TIs. For the mpASL for the first section, the minimum TI was $300 \mathrm{~ms}$ and subsequent TIs were increased by $250 \mathrm{~ms}$, label thickness was $130 \mathrm{~mm}$, and label gap was $20 \mathrm{~mm}$. A total of 2880 images, including 1440 labeled and 1440 control images, were obtained. For the spASL, the pulse delay time was $1200 \mathrm{~ms}$, label thickness was $100 \mathrm{~mm}$, and label gap was $20 \mathrm{~mm}$. In both spASL and mpASL, the labeling slab was positioned at the level of upper cervical region. Other features of the perfusion sequences are shown in the Table 1.

\section{Data Processing}

Image analysis was performed in an imaging workspace (Extended MR WorkSpace, Version 2.6.3.5; Philips Healthcare) with the special application tools, namely "neuro perfusion" and "Image Algebra" for DSC-PI and ASL-PI, respectively. DSC and ASL perfusion maps were evaluated by 2 radiologists with 4 and 20 years of neuroradiology experience (H.M. and Y.Ö.). Images were first evaluated by a radiologist (H.M.) with 4 years of neuroradiology experience. In cases where the first radiologist (H. M.) doubts, both radiologists evaluated the images together and arrived at the final decision. In addition, in the quantitative evaluation, the interrater agreement was evaluated using measurements from the ASL and DSC maps in another session. The same cutoff values were used in terms of benign-malignant distinction for a more objective evaluation. For the quantitative analysis, the lesions that had not previously undergone an operation and had no bleeding or calcification within the mass were classified as group 1 . The lesions that had previous surgical treatment or had bleeding and/or calcifications within the mass were classified as group 2. In another session, lesions with both single- and multiphase ASL examinations were assessed by a qualitative analysis for malignancy detection.

\section{Evaluation of ASL Perfusion}

In the ASL data processing, subtraction images of the labeled and control images were obtained. The technique of generating 


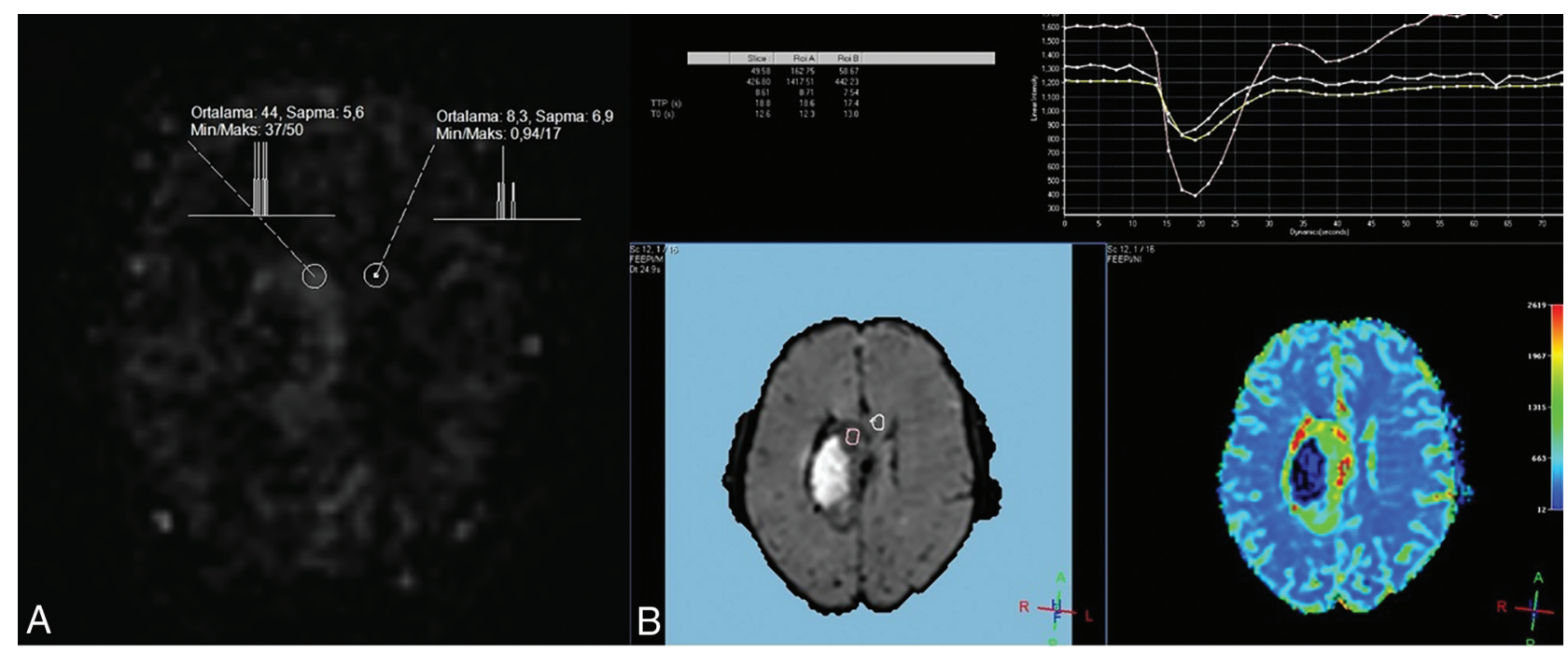

FIG 1. ROls were located in the tumor and white matter of the contralateral hemisphere, and the measurements are seen on mpASL $(A)$ and DSC (B) perfusion maps.

pulsed single- and multiphase ASL perfusion maps is described elsewhere. ${ }^{11-14}$ Typically, the spASL measurements were obtained at a single TI between labeling and image acquisition. In the mpASL mapping, for the first slice, minimum inversion time was $300 \mathrm{~ms}$, and subsequent inversion times were increased by 250 ms, and 48 subtraction images of the labeled and control images were obtained. The tumors were evaluated in the image in which delineation was best observed.

For the quantitative analysis in mpASL, a manually drawn elliptic ROI was placed on the solid and brightest portion of tumor seen in the subtraction images, which was assumed to have a high rate of perfusion. On average, 3 ROIs were placed on the basis of the lesion size, and the highest rCBF values were chosen. To normalize the values obtained, we drew an ROI of a size equal to the white matter in the contralateral hemisphere, which was the mirror symmetry of the tumor localization. A large ROI from the contralateral centrum semiovale was placed for patients in whom the ROI in the contralateral white matter could be small or problematic. The value of the maximum relative $\mathrm{CBF}(\mathrm{rCBF})$ of the tumor divided by the $\mathrm{rCBF}$ of the contralateral normal WM provided the value of the $\operatorname{rrCBF}$ (Fig 1). We used conventional sequences to place the ROI on the solid part of the tumor on the perfusion map. In particular, we used the T2-weighted images.

In the qualitative evaluation, the scoring was performed on a scale from 0 to 3 . A zero score indicated that the lesion was hypointense compared with the white matter. A score of 1 indicated an isointense lesion compared with the white matter, and a score of 2 indicated a hyperintense lesion compared with the white matter. A score of 3 indicated that the lesion was remarkably hyperintense. The lesions with scores of 0 and 1 were considered benign, whereas the lesions with scores of 2 and 3 were considered malignant.

\section{Evaluation of DSC Perfusion}

For the quantitative evaluation, the arterial input function was determined after selecting the anterior cerebral artery or the middle cerebral artery on draft images to create DSC perfusion maps, marking the contrast plateau on signal intensity-time curves, and finally creating the color maps. The contrast agent (gadolinium) that is extravasated due to the impaired blood-brain barrier causes permanent signal loss on T2-weighted sequences due to residual effect. Permanent signal loss influences first-passage images and subsequent recirculated images. ${ }^{15}$ To prevent the occurrence of leaking artifacts, software and correction algorithms were used (Extended MR WorkSpace, Version 2.6.3.5). The lesion was detected on the color DSC perfusion map, and relative regional $\mathrm{CBF}$ and $\mathrm{CBV}$ values were calculated by repeating the above-mentioned procedures with the ASL technique (Fig 1).

\section{Statistical Analysis}

The SPSS 23.0 program (IBM, Armonk, New York) was used for the statistical analyses. Receiver operating characteristic (ROC) curve analysis was used for determining the respective cutoff values. Comparison of ROC curves was performed by MedCalc, Version 18.11.0 (MedCalc Software, Mariakerke, Belgium). Normality tests were used for determining the distribution of continuous data (Kolmogorov-Smirnov), and nonparametric tests were used. The correlation between the perfusion parameters was assessed using the Spearman correlation analysis. The $\kappa$ test was used for interrater agreement. $P<.05$ was considered significant.

\section{RESULTS}

A total of 86 lesions in 79 patients were evaluated. Of the included lesions, 48 (55.8\%) were in men and 38 (44.2\%) were in women, with a mean age of $48.9 \pm 18.6$ years. The diagnosis was made histopathologically in $38(44.2 \%)$ lesions. The remaining 48 (55.8\%) lesions were diagnosed during the follow-up period. Of the lesions in which the diagnosis was made histopathologically, 14 were glioblastomas, 7 were diffuse astrocytomas, 7 were anaplastic astrocytomas, 2 were WHO grade II oligodendrogliomas, 2 were anaplastic oligondendrogliomas, 2 were gliosarcomas, 2 
Table 2: Histopathologic and follow-up diagnosis

\begin{tabular}{lcc}
\hline & No. & Percentage \\
\hline Histopathologic diagnosis & 38 & 44.2 \\
Benign lesions & 13 & 15.1 \\
Diffuse astrocytoma & 7 & 8.1 \\
WHO grade II oligodendroglioma & 2 & 2.3 \\
DNET & 2 & 2.3 \\
Cerebellar astrocytoma & 1 & 1.2 \\
Ganglioglioma & 1 & 1.2 \\
Malignant lesions & 25 & 29.1 \\
Glioblastoma & 14 & 16.3 \\
Anaplastic astrocytoma & 7 & 8.1 \\
Anaplastic oligondendroglioma & 2 & 2.3 \\
Gliosarcoma & 2 & 2.3 \\
Follow-up diagnosis & 48 & 55.8 \\
Benign lesions & 32 & 37.2 \\
Malignant lesions & 16 & 18.6 \\
Total & 86 & 100 \\
\hline
\end{tabular}

Note:-DNET indicate dysembryoplastic neuroepithelial tumors.

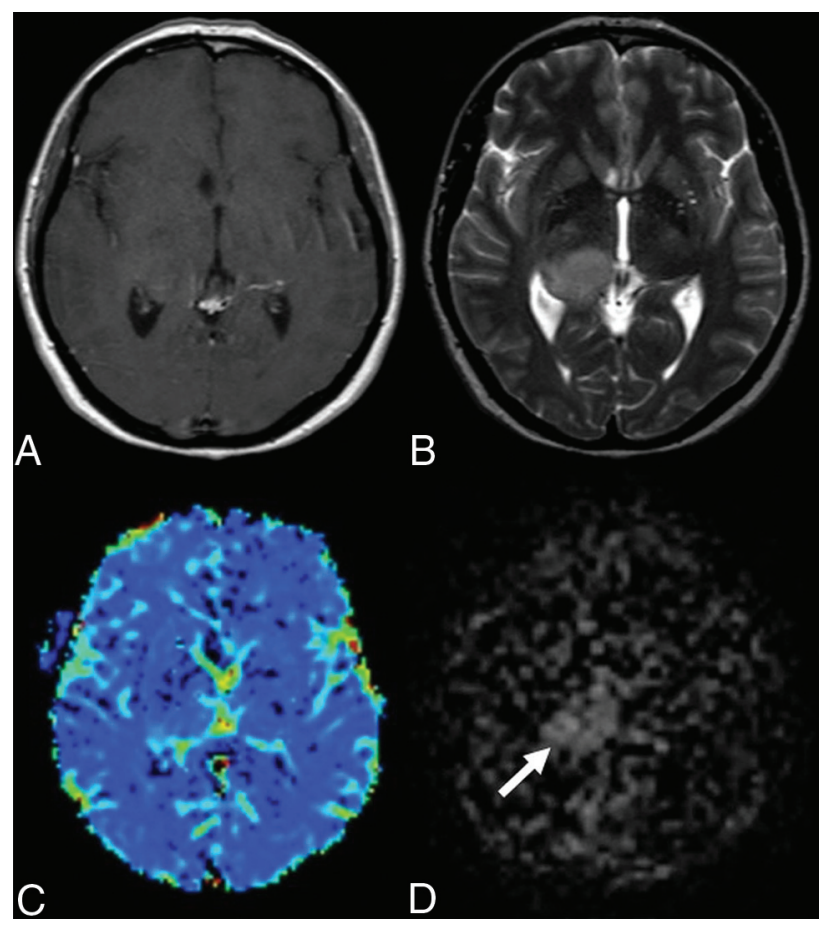

FIG 2. Anaplastic astrocytoma in a 44-year-old female patient. There is a mass in the T2-weighted $(B)$ sequence showing a signal increase in the right thalamus. Also, there is a slight expansion in both T2-weighted $(B)$ and the postcontrast T1-weighted $(A)$ sequences. There is no significant enhancement in the postcontrast T1-weighted $(A)$ MR image. There are no significant increases in perfusion in the mass localization derived from the DSC (C) perfusion color map (DSC $\mathrm{rrCBF}=1.1$ ). In the mpASL perfusion map $(D)$, the perfusion increase in the mass localization can be seen clearly (ASL rrCBF = 3.3) (arrow).

were dysembryoplastic neuroepithelial tumors, 1 was a cerebellar astrocytoma, and 1 was a ganglioglioma (Table 2).

In 48 lesions, the diagnosis was made by follow-up imaging (32 benign lesions, 16 malignant lesions). Sixteen lesions were diagnosed as malignant due to an increase in the lesion size and/ or increased enhancement/perfusion during follow-up (Table 2). The mean follow-up period was 20.18 months (range, 11-60 months).
There were 38 lesions in group 1 (patients underwent no previous operations and had no findings of intratumoral hemorrhage/calcification) (Fig 2). Lesions with intratumoral hemorrhages and/or calcifications and lesions having undergone an operation previously for brain tumors (48 cases total) were recorded as group 2 (Fig 3).

The mean $\mathrm{rCBF}$ and $\mathrm{rCBV}$ values were approximately $2.3-$ 2.7 times higher in the lesions with malignant primary tumors compared with the lesions with benign primary tumors, using the combination of histopathology or 11-month stability. There was a significant difference between the malignant and benign groups in terms of the both $\mathrm{rrCBF}$ and $\mathrm{rrCBV}$ values $(P<.001$ for both values and technique) (Table 3 ).

In the ROC analysis performed for evaluating the perfusion characteristics, the areas under the curve for $\mathrm{rrCBF}$ in the mpASL and DSC were found to be 0.879 and 0.886 , respectively (Fig 4). On the basis of equal misclassification rates, cutoff values of 1.4 and 1.5, respectively, for the mpASL $\operatorname{rrCBF}$ and DSC $\mathrm{rrCBF}$ ratios, were the best in discriminating benign from malignant tumors (Table 3). For the quantitative evaluation, rrCBV measurements were also performed with the DSC-PI. In the ROC analysis, the area under the curve for perfusion evaluation of the rrCBV was calculated to be 0.902 (Fig 4). A cutoff value of 2.2 was accepted for the rrCBV value in DSC-PI (Table 3). No statistically significant difference was found in the comparison of ROC curves in group 1 , in group 2 , and in all lesions ( $P$ values between .214 and .821$)$.

The interrater agreement was good for ASL map $(\kappa=0.766)$ and very good for CBF and CBV on the DSC map $(\kappa=0.907$ and $\kappa=0.923$, respectively). The correlation analyses among the perfusion parameters revealed a strong correlation between the mpASL $\operatorname{rrCBF}$ and the DSC $\operatorname{rrCBF}(r=713, P<.001)$. There was also a strong correlation between the mpASL rrCBF and the DSC $\operatorname{rrCBV}(r=675, P<.001)$. Furthermore, there was a very strong correlation between the DSC $\mathrm{rCBF}$ and the DSC $\mathrm{rrCBV}$ $(r=907, P<.001)$.

There were 55 lesions (26 benign and 29 malignant lesions) that had undergone both single and multiphase ASL-PI. In the qualitative evaluation of the mpASL-PI map, 23 of 29 malignant lesions were diagnosed correctly and all 26 benign lesions were diagnosed correctly (mpASL-PI, sensitivity $=79.3 \%$, specificity $=100 \%)$. On the qualitative evaluation of the spASL-PI map, 14 of 29 malignant lesions were diagnosed correctly and all 26 benign lesions were diagnosed correctly (spASL-PI: sensitivity = $48.2 \%$, specificity $=100 \%$ ). Although there was a perfusion increase in the mpASL in 9 malignant cases, there was no increase in perfusion was observed in the spASL imaging (Fig 5).

\section{DISCUSSION}

In our study, we found that the rCBF values in both mpASL and DSC perfusion MR imaging were more sensitive than the $\mathrm{rCBV}$ values in DSC perfusion MR imaging in identifying the tumor tissue in the lesions with susceptibility artifacts due to bleeding and/ or calcifications. When the mpASL and DSC perfusion MR imaging were compared, the sensitivity and accuracy of ASL perfusion was higher but the difference was not statistically significant. These findings are consistent with the literature. Previously, the 
superiority of mpASL to spASL was shown in healthy patients. ${ }^{16,17}$ In our study, we also showed that mpASL was significantly superior in patients with primary brain tumors.

In the literature, there are many studies comparing the functional and conventional MR imaging techniques, showing that rrCBV is the most sensitive marker in differentiating benign from malignant lesions and in evaluating whether a brain tumor is recurrent or residual. ${ }^{14,18-21}$ However, most of these studies did not use ASL-PI. ${ }^{3,4}$ In most studies using ASL-PI, only spASL was evaluated, ${ }^{18-20}$ and some studies reported that spASL had a higher

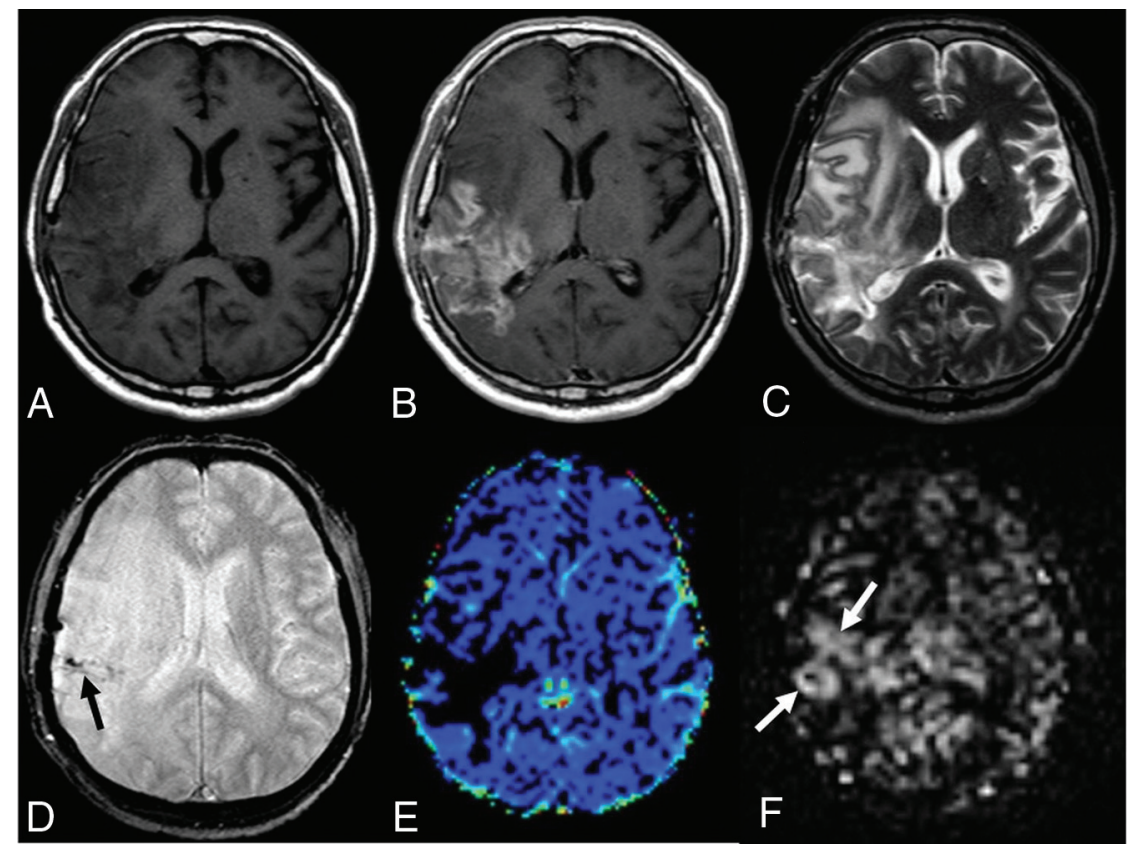

FIG 3. A 49-year-old male patient who underwent an operation and received chemoradiotherapy due to a glioblastoma in the temporoparietal region 1 year ago. T1-weighted $(A)$ and T2-weighted (C) MR images show postoperative changes and alterations in the signal intensities in the right temporoparietal region. The postcontrast image $(B)$ shows wide-scale enhancement in the site of the operation. The $2^{*}$-weighted image $(D)$ shows hypointensities secondary to old bleeding residues (black arrow). The DSC perfusion map $(E)$ shows a large perfusion signal loss caused by leakage artifacts in the contrasted areas (DSC $\operatorname{rrCBF}=1.3$ ). In the mpASL perfusion map $(F)$, there is a significant increase in perfusion at the site of the operation (white arrows) (ASL rrCBF = 3.5). The patient was diagnosed with a recurrent glioblastoma after the second operation. sensitivity. ${ }^{14}$ Ozsunar et $\mathrm{al}^{14}$ showed that the sensitivity of ASL was high (94\%), but its specificity was low (50\%) in a study comparing spASL with DSC-PI in distinguishing tumor recurrence from radiation necrosis. Cebeci et $\mathrm{al}^{21}$ conducted a study and compared mpASL and DSC perfusion techniques, reporting that the specificity of ASL was higher than that obtained in DSC perfusion $(92.3 \%$ versus $76.9 \%$ ). When ASL and DSC perfusion were used in combination, all malignant lesions were diagnosed accurately. In our study, we found that the rCBF value derived from mpASL perfusion had a higher sensitivity and accuracy compared with the DSC perfusion parameters, $\mathrm{rCBF}$ and $\mathrm{rCBV}$, in discriminating benign and malignant tumors. However, this difference was not statistically significant. Although the specificity of rrCBF values in DSC perfusion was slightly lower, the sensitivity and accuracy rates were higher than those of rrCBV.

In the literature, it has been reported that intratumoral calcifications and/or bleeding or postoperative changes cause susceptibility artifacts more commonly in DSC compared with ASL. ${ }^{9-11}$ In pathologies in which the blood-brain barrier is severely impaired (such as in glioblastomas and meningiomas), the leakage of the contrast medium into the extravascular area is one of the reasons for incorrect calculations in DSC-PI. ${ }^{22}$ However, the studies in this field are limited and not systematic. In the current study, which examined 2 groups of lesions (with native tumors and with tumors that might contain artifacts), we found that susceptibility artifacts resulted in a decrease in the diagnostic sensitivity in both the mpASL-PI and DSC-PI methods at similar rates (in group 2, both ASL and DSC sensitivity was $80 \%)$. This situation may be related

Table 3: Perfusion parameters of benign and malignant tumors on the quantitative evaluation

\begin{tabular}{|c|c|c|c|c|c|c|}
\hline & $\begin{array}{c}\text { Benign Tumors } \\
\text { Mean }( \pm S D)\end{array}$ & $\begin{array}{c}\text { Malignant Tumors } \\
\text { Mean ( } \pm \text { SD) }\end{array}$ & $P^{a}$ & $\begin{array}{c}\text { Sensitivity } \\
(\%)\end{array}$ & $\begin{array}{c}\text { Specificity } \\
(\%)\end{array}$ & $\begin{array}{c}\text { Accuracy } \\
(\%)\end{array}$ \\
\hline \multicolumn{7}{|c|}{ All lesions $(n=86)^{b}$} \\
\hline mpASL rrCBF & $1.19(0.59)$ & $2.84(1.44)$ & $<.001$ & 85.4 & 84.4 & 84.9 \\
\hline DSC rrCBF & $1.19(0.69)$ & $2.93(1.57)$ & $<.001$ & 82.9 & 80.0 & 81.45 \\
\hline DSC rrCBV & $1.20(0.62)$ & 3.07 (1.43) & $<.001$ & 68.3 & 97.8 & 83.05 \\
\hline \multicolumn{7}{|c|}{ Group $1(n=38)^{c}$} \\
\hline mpASL rrCBF & $1.24(0.47)$ & $3.06(1.45)$ & $<.001$ & 94.4 & 80.0 & 87.2 \\
\hline DSC rrCBF & $1.33(0.83)$ & $3.13(1.80)$ & $<.001$ & 88.9 & 75.0 & 81.95 \\
\hline DSC rrCBV & $1.32(0.67)$ & $3.22(1.43)$ & $<.001$ & 66.7 & 100 & 83.35 \\
\hline \multicolumn{7}{|c|}{ Group $2(n=48)^{d}$} \\
\hline mpASL rrCBF & $1.16(0.68)$ & $2.67(1.45)$ & $<.001$ & 78.3 & 88.0 & 83.15 \\
\hline DSC rrCBF & $1.08(0.54)$ & $2.77(1.39)$ & $<.001$ & 78.3 & 84.0 & 81.15 \\
\hline DSC rrCBV & $1.10(0.58)$ & $2.96(1.45)$ & $<.001$ & 69.6 & 96.0 & 82.8 \\
\hline
\end{tabular}

\footnotetext{
${ }^{a}$ Mann-Whitney $U$ test.

${ }^{\mathrm{b}}$ Benign ( $\left.n=45\right)$, malignant $(n=41)$.

${ }^{c}$ Benign $(n=20)$, malignant $(n=18)$.
}

${ }^{\mathrm{d}}$ Benign ( $\left.n=25\right)$, malignant $(n=23)$. 


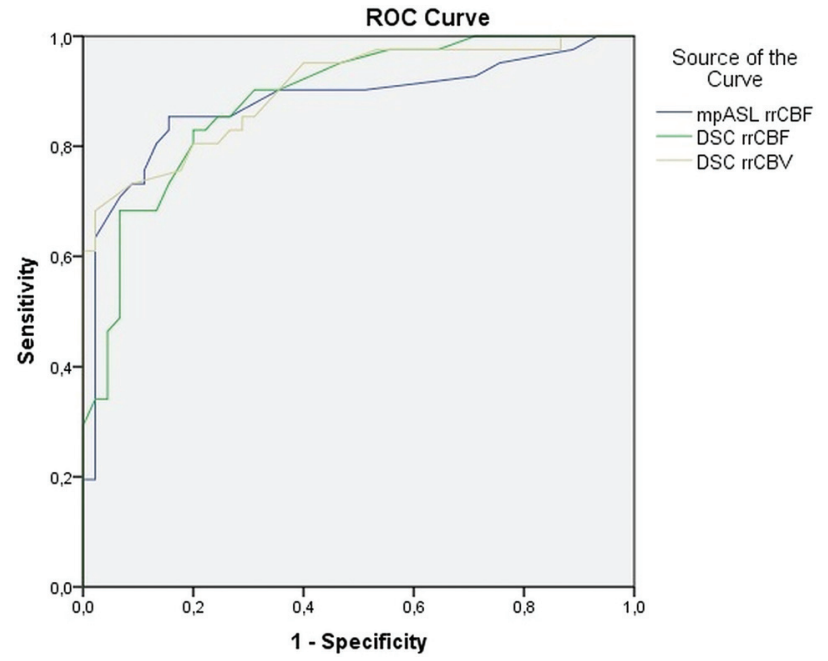

FIG 4. The ROC analysis curve. The blue line shows mpASL perfusion, and the green and the brown lines represent the DSC perfusion MR imaging technique for $\mathrm{rrCBF}$ and $\mathrm{rCBV}$, respectively.

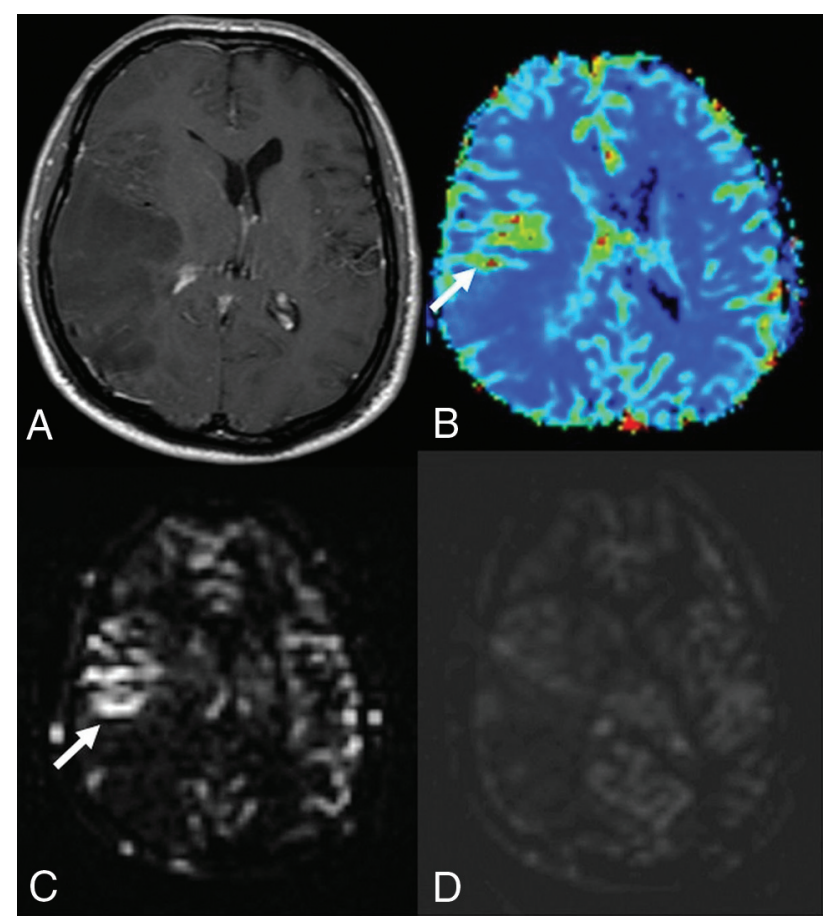

FIG 5. Anaplastic astrocytoma in a 41-year-old male patient. Postcontrast Tl-weighted $(A)$ image shows a wide nonenhancing mass lesion in the right temporoparietal region. There was no diffusion restriction (not shown here). The DSC $(B)$ and mpASL $(C)$ maps show significantly hyperperfused areas within the mass (arrows) (DSC $\operatorname{rrCBF}=2.3, \mathrm{ASL} r \mathrm{rCBF}=4.6$ ). The spASL $(D)$ map shows no increases in perfusion. It is also remarkable that the signal-to-noise ratio of spASL is lower than that of mpASL.

to the mpASL technique we use. However, the specificity was higher in group 2 (82.6\% and $78.3 \%$ in group $1,88.5 \%$ and $84.6 \%$ in group 2, respectively for mpASL and DSC). Signal loss due to hypoperfusion, microhemorrhages, and old bleeding residues secondary to an operation and radiation therapy may explain the lower false-positive values in group $2 .^{23}$
The recent 2016 World Health Organization classification has also shown us that genetic and molecular typing is crucial to progression in brain tumors. ${ }^{6}$ The minimum follow-up period in our study was 11 months. In some studies in the literature, the follow-up period was between 6 months and 2 years. ${ }^{5}$ Some previous studies that used a 6-month follow-up period were conducted before the relationship between some molecular markers and tumor prognosis was known. ${ }^{5}$

The disadvantages of the ASL perfusion technique are that only CBF mapping can be performed and $\mathrm{CBF}$ values can be measured. DSC perfusion can provide other parameters as well. Not obtaining other parameters, primarily the CBV, is an important limitation of the ASL technique. Although there are studies of the measurement of CBV by the ASL technique, the evaluation of ASL with CBV has not yet been used in clinical practice. $^{24}$ Moreover, geometric resolution of ASL perfusion maps obtained without contrast agent administration is lower than in the DSC perfusion maps, and low SNR is the other disadvantage of the ASL-PI. This problem may be overcome by the $3 \mathrm{D}$ pseudocontinuous method. The problem is less for examinations with high magnetic field strength. In our study, we used the pulsed ASL method on a $1.5 \mathrm{~T}$ device. The ASL technique needs multiple repetitions for higher SNR, which causes longer imaging times. ASL image acquisition at multiple sections at multiple time points was also an advantage of this study.

There are many options in using the ASL technique, namely the single-phase and multiphase, pulsed, and continuous or pseudocontinuous methods. ${ }^{23}$ There is no consensus on the clinical usefulness and diagnostic sensitivity of these methods in the literature. The spASL measurements are conducted at a single TI between labeling and image acquisition. The effects of the arterial arrival time or arterial transit time on CBF estimation make it difficult to get the best enhancement of the signal and evaluation. This problem can cause misinterpretation and errors in interpreting the qualitative and quantitative analyses of perfusion characteristics. Performing multiple ASL acquisitions at various TIs between the labeling and image acquisition can be used for solving this transit time problem. In our study, 63 lesions were qualitatively evaluated with multiphase and single-phase ASL techniques. In the qualitative evaluation with the mpASL-PI, while 27 of 34 malignant lesions were diagnosed correctly (sensitivity $=79.4 \%$ ), only 17 malignant lesions were diagnosed correctly on the spASL-PI (sensitivity $=50 \%$ ). As far as we know, there are no available studies comparing these 3 ASL methods in patients with brain tumors. However, in some studies, it has been shown that mpASL can give better SNR and more accurate CBF values in healthy subjects. ${ }^{16,17}$ We used the pulsed ASL at $1.5 \mathrm{~T}$ in our study. However, these studies use the pseudocontinuous ASL at $3 \mathrm{~T}^{16,17}$ Therefore, it is not possible to make a complete comparison between the results of pseudocontinuous ASL and pulsed ASL studies. On the basis of our findings and the data in the literature, we concluded that it would be more appropriate to use mpASL instead of spASL in terms of diagnostic reliability in the evaluation of patients with intracranial tumors.

Limitations of our study are the retrospective nature of the study design and the lack of availability of histopathologic diagnoses in all lesions. The use of a $1.5 \mathrm{~T}$ device in the study might 
have an unfavorable effect on sensitivity. Optimum pulse-labeling duration differs in different age groups, and this might affect the signal. mpASL can be used to overcome this potential pitfall. Vendors suggest the use of low-resolution mpASL to choose the optimum higher resolution spASL. We used this method as suggested at the beginning, but unfortunately, we could not apply it to all patients because we had a large number of patients and heavy workload. Therefore, we preferred to evaluate the 2 ASL methods separately in our study. This is one of the limitations of our methodology.

\section{CONCLUSIONS}

The sensitivity and specificity of mpASL are similar to those of DSC perfusion, irrespective of bleeding and calcification in primary brain tumors. Other advantages of ASL include it being a contrast-free method, repeatable, and less costly. Therefore, we believe that noncontrast multiphase ASL can be used instead of DSC MR perfusion in the diagnosis and follow-up of primary intracranial tumors.

\section{REFERENCES}

1. Kimura M, da Cruz LC Jr. Multiparametric MR imaging in the assessment of brain tumors. Magn Reson Imaging Clin $\mathrm{N} \mathrm{Am}$ 2016;24:87-122 CrossRef Medline

2. Heo YJ, Kim HS, Park JE, et al. Uninterpretable dynamic susceptibility contrast-enhanced perfusion MR images in patients with post-treatment glioblastomas: cross-validation of alternative imaging options. PLoS One 2015;10:e0136380 CrossRef Medline

3. Law M, Yang S, Wang H, et al. Glioma grading: sensitivity, specificity, and predictive values of perfusion $M R$ imaging and proton MR spectroscopic imaging compared with conventional MR imaging. AJNR Am J Neuroradiol 2003;24:1989-98 Medline

4. Knopp EA, Cha S, Johnson G, et al. Glial neoplasms: dynamic contrast-enhanced T2*-weighted MR imaging. Radiology 1999;211:79198 CrossRef Medline

5. Ata ES, Turgut M, Eraslan C, et al. Comparison between dynamic susceptibility contrast magnetic resonance imaging and arterial spin labeling techniques in distinguishing malignant from benign brain tumors. Eur J Radiol 2016;85:1545-53 CrossRef Medline

6. Louis DN, Perry A, Reifenberger G, et al. The 2016 World Health Organization Classification of Tumors of the Central Nervous System: a summary. Acta Neuropathol 2016;131:803-20 CrossRef Medline

7. Hu LS, Baxter LC, Smith KA, et al. Relative cerebral blood volume values to differentiate high-grade glioma recurrence from posttreatment radiation effect: direct correlation between image-guided tissue histopathology and localized dynamic susceptibility-weighted contrast-enhanced perfusion MR imaging measurements. AJNR Am J Neuroradiol 2009;30:552-58 CrossRef Medline

8. Aksoy FG, Lev MH. Dynamic contrast-enhanced brain perfusion imaging: technique and clinical applications. Semin Ultrasound CT MR 2000;21:462-77 CrossRef Medline

9. Lev MH, Ozsunar Y, Henson JW, et al. Glial tumor grading and outcome prediction using dynamic spin-echo MR susceptibility mapping compared with conventional contrast-enhanced
MR: confounding effect of elevated rCBV of oligodendrogliomas [corrected]. AJNR Am J Neuroradiol 2004;25:214-21 Medline

10. Xu Q, Liu Q, Ge H, et al. Tumor recurrence versus treatment effects in glioma: a comparative study of three-dimensional pseudo-continuous arterial spin labeling and dynamic susceptibility contrast imaging. Medicine (Baltimore) 2017;96:e9332 CrossRef Medline

11. Kimura H, Takeuchi H, Koshimoto $\mathrm{Y}$, et al. Perfusion imaging of meningioma by using continuous arterial spin-labeling: comparison with dynamic susceptibility-weighted contrast-enhanced MR images and histopathologic features. AJNR Am J Neuroradiol 2006;27:85-93 Medline

12. Warmuth C, Gunther M, Zimmer C. Quantification of blood flow in brain tumors: comparison of arterial spin labeling and dynamic susceptibility-weighted contrast-enhanced MR imaging. Radiology 2003;228:523-32 CrossRef Medline

13. Weber MA, Thilmann C, Lichy MP, et al. Assessment of irradiated brain metastases by means of arterial spin-labeling and dynamic susceptibility-weighted contrast-enhanced perfusion MRI: initial results. Invest Radiol 2004;39:277-87 CrossRef Medline

14. Ozsunar Y, Mullins ME, Kwong K, et al. Glioma recurrence versus radiation necrosis? A pilot comparison of arterial spin-labeled, dynamic susceptibility contrast enhanced MRI, and FDG-PET imaging. Acad Radiol 2010;17:282-90 CrossRef Medline

15. Asllani I, Borogovac A, Brown TR. Regression algorithm correcting for partial volume effects in arterial spin-labeling MRI. Magn Reson Med 2008;60:1362-71 CrossRef Medline

16. Fazlollahi A, Bourgeat P, Liang X, et al. Reproducibility of multiphase pseudo-continuous arterial spin labeling and the effect of post-processing analysis methods. Neuroimage 2015;117:191-201 CrossRef Medline

17. $\mathrm{Wu} \mathrm{B}, \mathrm{Lou} \mathrm{X}, \mathrm{Wu} \mathrm{X}$, et al. Intra- and interscanner reliability and reproducibility of $3 \mathrm{D}$ whole-brain pseudo-continuous arterial spin-labeling MR perfusion at 3T. J Magn Reson Imaging 2014;39:402-09 CrossRef Medline

18. Ma H, Wang $\mathrm{Z}, \mathrm{Xu} \mathrm{K}$, et al. Three-dimensional arterial spin labeling imaging and dynamic susceptibility contrast perfusionweighted imaging value in diagnosing glioma grade prior to surgery. Exp Ther Med 2017;13:2691-98 CrossRef Medline

19. Choi YJ, Kim HS, Jahng GH, et al. Pseudoprogression in patients with glioblastoma: added value of arterial spin labeling to dynamic susceptibility contrast perfusion MR imaging. Acta Radiol 2013;54:448-54 CrossRef Medline

20. Seeger A, Braun C, Skardelly M, et al. Comparison of three different MR perfusion techniques and MR spectroscopy for multiparametric assessment in distinguishing recurrent high-grade gliomas from stable disease. Acad Radiol 2013;20:1557-65 CrossRef Medline

21. Cebeci H, Aydin O, Ozturk-Isik E, et al. Assessment of perfusion in glial tumors with arterial spin labeling; comparison with dynamic susceptibility contrast method. Eur J Radiol 2014;83:1914-19 CrossRef Medline

22. Boxerman JL, Schmainda KM, Weisskoff RM. Relative cerebral blood volume maps corrected for contrast agent extravasation significantly correlate with glioma tumor grade, whereas uncorrected maps do not. AJNR Am J Neuroradiol 2006;27:859-67 Medline

23. Pollock JM, Tan H, Kraft RA, et al. Arterial spin-labeled MR perfusion imaging: clinical applications. Magn Reson Imaging Clin N Am 2009;17:315-38 CrossRef Medline

24. Yan L, Li C, Kilroy E, et al. Quantification of arterial cerebral blood volume using multiphase-balanced SSFP-based ASL. Magn Reson Med 2012;68:130-39 CrossRef Medline 